\title{
Incremental View on Intelligence and High Intrinsic Motivation Increase Working Memory Training Compliance
}

\author{
ALVA APPELGREN ${ }^{1 *}$, SARA L. BENGTSSON ${ }^{1}$ and STINA SÖDERQVIST ${ }^{2}$ \\ ${ }^{1}$ Department of Clinical Neuroscience, Karolinska Institutet, Stockholm, Sweden \\ ${ }^{2}$ Cogmed Pearson Assessment, Sweden
}

\begin{abstract}
Summary: Working memory (WM) training is demanding both regarding time and cognitive endurance. Many participants who could benefit from completing the training lose their motivation to do so. Hence, it is valuable to address compliance with the training protocol from a motivational angle. Studies have shown that subjective views on intelligence influence motivation, where individuals believing that intelligence can increase with training, that is, an incremental mind-set, tend to try harder after setbacks and that high-intrinsic motivation relates to higher academic performance. We used questionnaires to measure the extent to which mind-set and intrinsic motivation influenced compliance to complete a WM training program of a minimum of 20 sessions of WM training. Only 53 out of 112 recruited participants, $(13$ years old $(S D=.61))$ completed the training. Our results showed that mindset and motivation significantly predicted compliance to training, with high motivation and incremental mind-set being associated with more completed sessions. Copyright @ 2015 John Wiley \& Sons, Ltd.
\end{abstract}

\section{INTRODUCTION}

Working memory (WM) is the ability to remember and use information for a shorter period, seconds. WM capacity is closely related to the ability to sustain attention and is a predictor of academic performance (Alloway \& Alloway, 2010; Bull, Espy, \& Wiebe, 2008; Gathercole, Brown, \& Pickering, 2003). The importance of WM for every-day functioning and academic performance has motivated research for developing methods to improve WM capacity with non-pharmacological interventions, such as computerized training programs (for review see Klingberg, 2010). An example of a computerized WM program used to train WM is Cogmed's WM training (Klingberg, Forssberg, \& Westerberg, 2002). This program consists of both visuospatial and verbal WM tasks. The software uses an adaptive level algorithm that adjusts the difficulty level after each trial to ensure that training is always performed on a level close to the trainee's highest capacity. This adaptive algorithm, together with the duration of the training, make cognitive training suitable for studying motivational aspects of persistence, such as how long a person is willing to continue practicing on a demanding level. This is important because several individuals, who would most likely benefit from the training, fail to complete the protocol. A recent training study that reported the number of participants who did not complete the protocol, found that $44 \%$ of the recruited children managed to perform 20 sessions, where each session was about 90 minutes (Jaeggi, Buschkuehl, Shah, \& Jonides, 2014).

\section{Intrinsic motivation}

It has been found that people are more willing to commit to goals that they have set up for themselves (Bandura, 1993). In an educational settings, a person's own will to do a task, that is their intrinsic motivation, has been evaluated in academic settings, where high-intrinsic motivation was

*Correspondence to: Alva Appelgren, Department of Clinical Neuroscience, Karolinska Institutet, Nobels väg 9, SE-17177 Stockholm, Sweden.

E-mail: alva.appelgren@gmail.com associated with learning and performance improvements (for review see Ryan \& Deci, 2000). For example, Vallerand, Fortier and Guay (1997) found that lower intrinsic motivation led to lower evaluations of the students' own competence and a greater urge to drop out from high school. Furthermore, children's belief in their own ability has been found to improve school performance (Miserandino, 1996). In their study, children who believed in their own ability reported more enjoyment and showed greater persistence in school tasks than children who felt uncertain about their ability. Furthermore, in a meta-analysis consisting of 18 studies on motivation, a positive association was found between intrinsic motivation and academic achievement (Taylor et al., 2014). Similarly, the goal of completing a WM training program and the belief in being able to do so may influence how much time one invests in training.

\section{Prior beliefs regarding intelligence}

People's beliefs regarding intelligence have been found to influence motivation and performance (Blackwell, Trzesniewski, \& Dweck, 2007; Dweck, Chiu, \& Hong, 1995; Dweck \& Leggett, 1988). Dweck (1986) has outlined two main distinctions in viewing intelligence; a person can have either an entity orientation, which means that this person views intelligence as something fixed, or an incremental view, meaning that this person regards intelligence as something changeable. It has been shown that people's mind-set regarding intelligence influences how they react to making mistakes. Individuals with an entity view are more likely to see mistakes as signs of their own inadequacy or inability (Dweck et al., 1995; Grant \& Dweck, 2003). On the other hand, individuals with an incremental view see mistakes as signals that they are working with difficult tasks that require further training to master. Aronson, Fried and Good (2002) conducted a study in which students were mentored so as to motivate an incremental mind-set. To encourage an incremental mind-set, students were taught that intelligence can be changed with mental work and that when facing difficulties, this is most likely due to the novelty of the situation 
rather than of someone's incapability. These students' performance was compared with a group of students who were taught about effects of drug use. The results showed that students receiving the incremental mind-set teaching style had greater math and reading progress compared with the control group. Furthermore, Blackwell et al. (2007) also found that an incremental mind-set was related to increased math grades compared with adolescents with an entity mind-set. A recent study using a computerized math test found that people exposed to an incremental mind-set intervention spent longer time with the task and completed more difficulty levels compared with a control group (O'Rourke, Haimovitz, Ballweber, Dweck, \& Popović, 2014). Furthermore, Moser, Schroder, Heeter, Moran and Lee (2011) found that people with an incremental mind-set showed greater accuracy improvements after mistakes compared with people with a more fixed mind-set. Taken together, these findings illustrate that an incremental mind-set can influence a person's behavior reflected in motivation and performance.

\section{The present study}

The aim of the present study was to investigate if mind-set and intrinsic motivation influence participants' (11-13 years old) compliance to a WM training protocol.We hypothesized that participants with an incremental mind-set and highintrinsic motivation would show higher compliance adhering to the WM training protocol, and thus, we predicted a positive correlation between motivation scores and number of training sessions completed, as well as a correlation between mind-set scores and number of training sessions completed.

We explored if the coaches for each school influenced the students' compliance with the training program and if the training at home or in school influenced the number of trained sessions.

\section{METHOD}

\section{Recruitment}

To recruit students, we sent out information regarding the study to the schools and teachers who were on Cogmed Coach mailing list, consisting of current Cogmed school customers in Sweden. After a coach (usually a teacher) had registered their interest for their class to participate, written information regarding the training and study was sent out to the coach. Subsequently, the coach distributed this information to the students and their legal guardians. Written consent for participation was required from both students and their legal guardian(s). The study was approved by the local ethics committee, Stockholm, Sweden (dnr 2013/1994-31/3).

\section{Participants}

One hundred and twelve adolescent students, 58 boys and 54 girls, mean age 13 years $(S D=0.61)$, from three different schools in Sweden signed up to participate in the study. Each school had one or two classes that signed up to participate, for which a majority of students in each class participated.

This study was part of a larger study investigating the effect of feedback given during the training. For this reason, participants within a class were pseudo-randomly (controlling for gender) assigned to one of the four feedback groups. These groups would receive different trial-based feedback during the training. Group 1 received encouraging feedback and two-tone sounds after correct responses. Group 2 received encouraging feedback and descending sounds after incorrect responses. Group 3 did not receive any feedback. Group 4 received sounds after correct and incorrect responses and encouraging and positive feedback. The results of the effect of feedback on training will be presented elsewhere.

A monetary reward of 5000 SEK was given to the class as a group if a minimum of 20 students completed the training. If less than 20 students completed the training, only a part of the sum calculated based on completed trainings was given to the class. Throughout the training, the coaches knew how many sessions the students had trained but were not instructed to communicate this to the class as a whole.

\section{Coaching}

Each school was assigned individual coaches. All coaches had participated in Cogmed's coach training. In this training, the coaches were instructed to support the students according to a set protocol. Thus, the coaches all received the same type of instructions that they were to give to their students. The coaches informed the participants individually on their progress and number of sessions trained. They were informed that the students would receive different feedback during training but did not know to which feedback group each participant belonged.

\section{Training procedure}

The participants performed the training at home or in school, which was a decision made by each school. Participants from School 1 trained at home, while participants from Schools 2 and 3 trained in school. Each training session lasted approximately $50 \mathrm{~min}$ and was performed using participants' or schools' computer or tablet. A minimum of 20 out of 25 training sessions was required for the training to be considered completed. The participants were allowed to take a break if there were school holidays or if they were absent from schools for other reasons, for example, illness. Therefore, the cut-off for each participant's number of total days to train was not fixed to the standard training period of five weeks but was extended in the cases where participants had absence from school.

\section{Training program}

Cogmed's WM training program is based on tasks developed in previous research (Klingberg et al., 2005; Klingberg et al., 2002). The software used was Cogmed's RM (Cogmed and Cogmed Working Memory Training are trademarks, in the USA and/or other countries, of Pearson Education, Inc. or its affiliate(s)), consists of visuo-spatial and verbal WM tasks. The tasks involve remembering the location and/or the order in which the stimuli (visual or verbal) were presented and responses are made by clicking/tapping on the items one at a time either in the order they are presented 
or in reverse order. The presentation time for each stimulus was $1000 \mathrm{msec}$. The time between each stimulus was 500 msec. Task difficulty is adjusted so that difficulty levels (the number of stimuli to be remembered) increase following correct responses and decrease following incorrect responses. The software recorded all the training results and the answers to the questions automatically.

\section{Questionnaires}

The questionnaires were filled in both before starting the WM training and again at the end of the training period. Only the participants who completed a minimum of 20 sessions responded to the questionnaire at the end of the training. Thus, only the questionnaires administered pretraining were used for this study. A person's intrinsic motivation can be measured with questions from the intrinsic motivation inventory (IMI) (McAuley, Duncan, \& Tammen, 1989; Ryan, 1982), which measures prior beliefs about a task and about one's own perceived ability to perform that task. On the first day of training, the participants answered six selected questions from the IMI (McAuley et al., 1989) and one in-house question. The original questionnaire contains 28 items with questions designed to measure intrinsic motivation in four areas; enjoyment, competence, effort, and pressure. McAuley et al., (1989) stated that a subset of the questions can be chosen and adjusted for the particular task at hand and this has been done by previous researchers studying exercise, motivation, and WM (Buckworth, Lee, Regan, Schneider, \& DiClemente, 2007; Söderqvist, Matsson, Peyrard-Janvid, Kere, \& Klingberg, 2014). We selected questions in the field of enjoyment, competence, and effort (Table 1) and adjusted them to fit WM training as the activity.

Mind-set is measured by theory of intelligence (TOI) statements with which participants either agree or disagree (Dweck et al., 1995). All participants answered three TOI

Table 1. Correlation of motivation scores and TOI scores with training duration. All correlations are reported and level of significance are displayed

\begin{tabular}{lc}
\hline Measures & $\begin{array}{c}\text { Sessions } \\
\text { completed }(r)\end{array}$ \\
\hline Motivation question average & $.23^{*}$ \\
Q1. I believe the training will be good for me & $.27^{* *}$ \\
Q2. I believe that I will be pretty good at this & -.07 \\
type of training & .16 \\
Q3. I will put effort into this when I do the training & -.05 \\
Q4. I believe the training will be challenging & $.27^{* *}$ \\
Q5. I believe the training will be fun & $.19^{*}$ \\
Q6. It is important for me to do well on this training & $.21^{*}$ \\
Q7. I believe I will go through with all of & $-.21^{*}$ \\
the training sessions & -.11 \\
TOI question average & $-.24^{*}$ \\
QI1. You have a certain amount of intelligence & \\
and you cannot do much to change it. & -.18 \\
QI2. Your intelligence is something that you & \\
cannot change very much. & \\
QI3. You can learn new things, but you cannot & \\
really change your basic intelligence & \\
\hline
\end{tabular}

Note: $* * p<.01, * p<.05$.

TOI, theory of intelligence. questions describing an entity orientation. These are presented in Table 1. In Dweck et al., (1995) participants answered these three questions with numbers on a 6-point scale. In our study, for consistency and to make it easier for the user, we used the same scale for both the motivation statements and TOI statements (range 1-7); $1=$ disagree completely, $4=$ neither agree nor disagree, and $7=$ completely agree. For the IMI statements, agreements (rating 5-7) represent highintrinsic motivation. For the TOI statements, agreement (rating 5-7) with the mind-set questions is consistent with an entity view, whereas disagreement (rating 1-3) is consistent with an incremental view.

\section{Statistical analysis}

We correlated average ratings on the three TOI questions and number of training sessions as well as average ratings on the seven motivation questions with the number of training sessions and reported significance at $p<.05$ level (two-tailed) using Pearson correlation in $R$ ( $\mathrm{R}$ 3.0.3). We also reported the correlations of the individual TOI and motivation questions using Spearman correlations. To measure if there was a difference in trained sessions depending on coach or if they trained at home or in school, we performed a one-way analysis of variance using completed training sessions as dependent variable and performing a post-hoc analysis using Tukey HSD tests.

\section{RESULTS}

\section{Training completion}

Fifty-three participants (24 boys and 29 girls) completed $\geq 20$ sessions of WM training, which represents $47 \%$ of the participants starting the experiment.

The average time to complete the training was 35.27 days $(S D=6.35)$, including school holidays. Because of technical failures, four participants did not fill in the questionnaires prior to training leaving 108 participants for the analysis including questionnaires.

\section{Mind-set, motivation, and sessions completed}

Mind-set scores (TOI-average) correlated negatively with number of sessions completed, $r(106)=-.21, p=.03$ (Table 1$)$. Thus, the more incremental mind-set (lower TOI scores) the more sessions were completed.

The average of the rating on the seven intrinsic motivation questions correlated positively with number of trained sessions, $r(106)=.23, p=.002$ (Table 1$)$. Four of these questions regarding intrinsic motivation correlated individually with number of trained sessions. These are marked with stars in Table 1.

\section{Coach and training duration}

In School 1, the 22 participants (14 boys and 8 girls), completed an average of $17.95(S D=8.65)$ sessions. In School 2 , the 66 participants ( 33 boys and 33 girls) completed an average of $13.67(S D=6.64)$ sessions. In School 3, the 24 participants (11 boys and 13 girls) completed an average of 22.3 
$(S D=6.03)$ sessions. There was a difference between number of completed sessions and schools, $F(2,109)=14.66$, $p=.001$, where the post-hoc analysis showed that School 2 differed significantly from the other schools $p<.05$ with less amount of completed sessions. Mind-set scores did not differ between schools $F(2,105)=1.83, p=.16$, nor did motivation scores $F(2,105)=2.49, p=.09$. There were no differences in number of completed training sessions depending on if the participants trained at home $(n=22)$ or in school $(n=90), F$ $(1,111)=1.17, p=.28$.

\section{DISCUSSION}

In this study, we investigated if prior beliefs about intelligence and intrinsic motivation influenced persistence to follow through with WM training. We found a negative correlation with mind-set scores and training sessions completed where an incremental mind-set was associated with greater compliance to completing the training. Previous research shows that an incremental mind-set makes people more willing to solve problems over a longer time (Blackwell et al., 2007; O'Rourke et al., 2014) and our results show that an incremental mind-set increases persistence to continue with demanding WM training in older childhood and adolescence. This points to a potentially important role for influencing participants' mind-set and attitudes prior to training in order to increase compliance and thereby positive effects following training interventions in line with encouraging incremental directed coaching (Rattan, Good, \& Dweck, 2012).

We also found that an average score of the subset of the IMI questions measuring subjective enjoyment, effort and competence correlated with completion of training. Our results show that if a participant had faith in their ability to perform the training and if he or she thought the training would be of value, the participant was more likely to complete a higher number of training sessions. In our study, the participants themselves had the choice to continue or quit the training and with a more optimistic view prior to the training, they persisted longer. This may be explained by the theory that people try to match their outcomes with their expectations (Dutton \& Brown, 1997).

Teacher's beliefs and instructions via mentoring can influence student's motivation (Aronson et al., 2002; Reeve, Jang, Carrell, Jeon, \& Barch, 2004). In fact, in the present study, the coach appeared to have an effect on training compliance. This finding was unexpected because the coaches had similar training and had been instructed to give similar support.

The type of response given by a coach when a student struggled with the training may have influenced the student's decision to either continue or quit the training. The impact of encouragements to students has been addressed previously (Rattan et al., 2012). Detailed examples on how to respond as a coach if the trainee is lacking motivation would be of great value in order to standardize the interactions between coaches and trainees. Although we lack documented data on the matter in our study, differences between coachers' instructions and support may explain why School 2 had fewer students who completed the training, because mind-set and motivation did not differ prior to WM training between the different schools.

\section{Limitation}

The differences in coaching style were unfortunately not analyzed because this was not a primary hypothesis for the study, and as a result, such information was not collected. More detailed instructions to the coaches should be taken into consideration for planning future studies.

Another limitation in the study was that the participants knew that after 20 participants had completed their training, monetary reward would be delivered to the class. This extrinsic reward may have influenced both the students' peer pressure to complete the training as well as the teachers' commitment to the participation. However, in cases where large proportion of the class dropped out, the class did not receive the monetary reward and it is therefore unlikely that the monetary reward was the reason why many children dropped out from School 2. If children dropped out from the training and told their peers, this may have influenced the compliance of the students who were still training, but we did not control for this issue.

We did not investigate the effect of socioeconomic status and engagement from parents, which may have influenced student's persistence in the training.

\section{Conclusion}

Here, we show that participants' expectations and prior beliefs regarding intelligence had an impact on compliance to a WM training protocol. This study sheds light on the importance of expectations and beliefs regarding intelligence in relation to learning and putting effort into improving WM capacity. Furthermore, the study illuminates the importance of support via coaching during training. Studying this in a highly demanding WM training is of great importance for the field of learning and education giving that a greater understanding of this can lead to improved design and implementation of future cognitive and educational interventions.

\section{CONFLICT OF INTEREST}

Stina Söderqvist is currently employed by Pearson, the distributors of Cogmed as used in this study. For the other authors, no conflict of interest declared.

\section{ACKNOWLEDGEMENTS}

The research was supported by the Swedish Research Council (VR), Cornell's Foundation, and Stockholm Brain Institute. We would like to thank the teachers, and adolescents who participated in this research. We would like to thank Kristina Svahnström for helping with recruitment of schools to participate. 


\section{REFERENCES}

Alloway, T. P., \& Alloway, R. G. (2010). Investigating the predictive roles of working memory and IQ in academic attainment. Journal of Experimental Child Psychology, 106(1), 20-9.DOI:10.1016/j.jecp.2009.11.003.

Aronson, J., Fried, C. B., \& Good, C. (2002). Reducing the effects of stereotype threat on African American college students by shaping theories of intelligence. Journal of Experimental Social Psychology, 38 (2), 113-125. DOI:10.1006/jesp.2001.1491.

Bandura, A. (1993). Percieved self-efficacy in cognitive development and functioning. Educational Psychologist, 28(2), 117-148.

Blackwell, L. S., Trzesniewski, K. H., \& Dweck, C. S. (2007). Implicit theories of intelligence predict achievement across an adolescent transition: A longitudinal study and an intervention. Child Development, 78 (1), 246-63. DOI:10.1111/j.1467-8624.2007.00995.x.

Buckworth, J., Lee, R. E., Regan, G., Schneider, L. K., \& DiClemente, C. C. (2007). Decomposing intrinsic and extrinsic motivation for exercise: Application to stages of motivational readiness. Psychology of Sport and Exercise, 8(4), 441-461.

Bull, R., Espy, K. A., \& Wiebe, S. (2008). Short-term memory, working memory, and executive functioning in preschoolers: longitudinal predictors of mathematical achievement at age 7 years. Developmental Neuropsychology, 33(3), 205-28. DOI:10.1080/87565640801982312.

Dutton, K., \& Brown, J. D. (1997). Global self-esteem and specific selfviews as determinants of people's reactions to success and failure. Journal of Personality and Social Psychology, 73(1), 139-148. DOI:10.1037//0022-3514.73.1.139.

Dweck, C. S. (1986). Motivational processes affecting learning. American Psychologist, 41(10), 1040-1048.

Dweck, C. S., Chiu, C., \& Hong, Y. (1995). Implicit theories and their role in judgments and reactions : A word from two perspectives author(s) inquiry. Readers must contact LEA for permission to implicit theories and their role in judgments and reactions : A world from two perspectives.

Dweck, C. S., \& Leggett, E. L. (1988). A social cognitive approach to motivation and personality. Psychological Review, 95(2), 256-273. DOI:10.1037//0033-295X.95.2.256

Gathercole, S. E., Brown, L., \& Pickering, S. J. (2003). Educational and child. Education and Child Psychology, 20(3), 109-122.

Grant, H., \& Dweck, C. S. (2003). Clarifying achievement goals and their impact. Journal of Personality and Social Psychology, 85(3), 541-53. DOI:10.1037/0022-3514.85.3.541.

Jaeggi, S. M., Buschkuehl, M., Shah, P., \& Jonides, J. (2014). The role of individual differences in cognitive training and transfer. Memory \& Cognition, 42(3), 464-80. DOI:10.3758/s13421-013-0364-z.

Klingberg, T. (2010). Training and plasticity of working memory. Trends in Cognitive Sciences, 14(7), 317-24. DOI:10.1016/j.tics.2010.05.002.

Klingberg, T., Fernell, E., Olesen, P. J., Johnson, M., Gustafsson, P., Dahlström, K., ... Westerberg, H. (2005). Computerized training of working memory in children with ADHD - a randomized, controlled trial. Journal of the American Academy of Child and Adolescent Psychiatry, 44(2), 177-86. DOI: 10.1097/00004583-200502000-00010

Klingberg, T., Forssberg, H., \& Westerberg, H. (2002). Training of working memory in children with ADHD. Journal of Clinical and Experimental Neuropsychology, 24(6), 781-91. DOI:10.1076/jcen.24.6.781.8395.

McAuley, E., Duncan, T., \& Tammen, V. V. (1989). Psychometric properties of the intrinsic motivation inventory in a competitive sport setting: a confirmatory factor analysis. Research Quarterly for Exercise and Sport, $60(1), 48-58$

Miserandino, M. (1996). Children who do well in school: Individual differences in perceived competence and autonomy in above-average children. Journal of Educational Psychology, 88(2), 203-214. DOI:10.1037/0022 0663.88.2.203.

Moser, J. S., Schroder, H. S., Heeter, C., Moran, T. P., \& Lee, Y.-H. (2011) Mind your errors: Evidence for a neural mechanism linking growth mindset to adaptive post-error adjustments. Psychological Science, 22(12), 1484-9. DOI:10.1177/0956797611419520.

O’Rourke, E., Haimovitz, K., Ballweber, C., Dweck, C., \& Popović, Z. (2014). Brain points: A growth mind-set incentive structure boosts persistence in an educational game. Proceedings of the 32nd Annual ACM Conference on Human Factors in Computing Systems - CHI '14, 3339-3348. DOI: $10.1145 / 2556288.2557157$

Rattan, A., Good, C., \& Dweck, C. S. (2012). "It's ok - not everyone can be good at math": Instructors with an entity theory comfort (and demotivate) students. Journal of Experimental Social Psychology, 48 (3), 731-737. DOI:10.1016/j.jesp.2011.12.012.

Reeve, J., Jang, H., Carrell, D., Jeon, S., \& Barch, J. (2004). Enhancing students' engagement by increasing teachers' autonomy support. Motivation and Emotion, 28(2), 147-169. DOI:10.1023/B MOEM.0000032312.95499.6f

Ryan, R., \& Deci, E. (2000). Intrinsic and extrinsic motivations: Classic definitions and new directions. Contemporary Educational Psychology, 25 (1), 54-67. DOI:10.1006/ceps.1999.1020.

Ryan, R. M. (1982). Control and information in the intrapersonal sphere: An extension of cognitive evaluation theory. Journal of Personality and Social Psychology, 43(3), 450-461. DOI:10.1037/0022-3514.43.3.450.

Söderqvist, S., Matsson, H., Peyrard-Janvid, M., Kere, J., \& Klingberg, T. (2014). Polymorphisms in the Dopamine Receptor 2 gene region Influence Improvements during Working Memory Training in Children and Adolescents. Journal of Cognitive Neuroscience, 26(1), 54-62.

Taylor, G., Jungert, T., Mageau, G., Schattke, K., Dedic, H., Rosenfield, S., \& Koestner, R. (2014). A self-determination theory approach to predicting school achievement over time: The unique role of intrinsic motivation. Contemporary Educational Psychology, 39(4), 342-358. DOI:10.1016/j.cedpsych.2014.08.002.

Vallerand, R. J., Fortier, M. S., \& Guay, F. (1997). Self-determination and persistence in a real-life setting: Toward a motivational model of high school dropout. Journal of Personality and Social Psychology, 72(5), $1161-76$. 